\title{
Von Nähe und Sicherheit: Die Artikel-Verordnung nach §§26, 27 Standortauswahlgesetz - Sicherheitsanforderungen, Dogmatik, Öffentlichkeitsbeteiligung
}

\author{
Ulrich Smeddinck
}

(c) Der/die Autor(en) 2021. Dieser Artikel ist eine Open-Access-Publikation.

\begin{abstract}
Die Standortsuche für ein Endlager ist neu angelaufen. Für die Auswahl von günstigen Teilgebieten wurden Sicherheitskriterien auf Geodaten angewendet. Regelungen zu Kriterien und zur Untersuchung von Sicherheitsaspekten sind insbesondere in der neuen Artikel-Verordnung nach SSS26, 27 StandAG geregelt. Der Beitrag informiert über die Regelungsinhalte, setzt aber den Schwerpunkt auf die Beteiligung der allgemeinen Öffentlichkeit im Verordnungsverfahren. Vor dem Hintergrund wird die Dogmatik der Beteiligung bei der Erarbeitung von Rechtsverordnungen hinterfragt. Die Bedeutung von Nähe und Transparenz werden als Leitwerte gegen Vertrauensverluste und Elitenverachtung konturiert.
\end{abstract}

\section{Einleitung}

Ein vermeintlich neutrales Sicherheitskriterium wurde zum Politikum als der bayerische Ministerpräsident Söder im Zuge des Verordnungsverfahrens intervenierte, um Granit als geeignetes Wirtsgestein für ein Endlager auszuschließen. ${ }^{1}$ Die Artikel-Verordnung über die Sicherheitsanforderungen und vorläufige Sicherheitsuntersuchungen für die Endlagerung hochradioaktiver $\mathrm{Abfälle}^{2}$ versammelt zwei Verordnungen: die Verordnung über Sicherheitsanforderungen an die Endlagerung hochradioaktiver Abfälle (Endlagersicherheitsanforderungsverordnung - EndlSiAnfV) und die Verordnung über Anforderungen an die Durchführung der vorläufigen Sicherheitsuntersuchungen im Standortauswahlverfahren für die Endlagerung hochradioaktiver Abfälle (Endlagersicherheitsuntersuchungsverordnung - EndlSiUntV). Sie sind nicht nur durch das Verordnungsverfahren verbunden, ${ }^{3}$ sondern auch inhaltlich aufeinander bezogen. Beide Verordnungen liefern wichtige Regelungen und Maßgaben auf dem Weg von der ,weißen Landkarte" ${ }^{4}$ zum konkreten Endlager und vervollständigen neben dem Geologiedatengesetz $z^{5}$ weiter die regulatorische Grundausstattung für die folgenden Verfahrensschritte zur Standortauswahl.

Anders als üblich wurden im Verfahren nicht nur die (Fach-)Kreise und Behörden involviert. Für die Verordnungsentwürfe wurde die Beteiligung einer allgemeinen Öffentlichkeit durchgeführt, die so rechtlich nicht vorgeschrieben und generell für Verordnungen bisher unüblich ist. Den Anstoß für diese Innovation - vor dem Hintergrund einer einschlägigen rechtwissenschaftlichen Diskussion über Bürgerbeteiligung in der ,Verordnungsgebung“" - gab die neue Kultur des ermächtigenden Standortauswahlgesetzes. ${ }^{7}$ Auch wenn es bereits zu den Sicherheitsanforderungen an die Endlagerung wärmeentwickelnder

Apl. Prof. Dr. Ulrich Smeddinck,

an der MLU Halle-Wittenberg,

Senior Researcher am Institut für Technikfolgenabschätzung

und Systemanalyse (ITAS/KIT), Karlsruhe,

Leiter des Teilprojektes DIPRO im BMWi-geförderten

TRANSENS-Forschungsverbund, Halle-Wittenberg, Deutschland radioaktiver Abfälle vom 30. September 2010 eine informelle Öffentlichkeitsbeteiligung auch für zivilgesellschaftliche Gruppen gab, ${ }^{8}$ bedeuten die Anstrengungen praktisch wie dogmatisch einen Quantensprung. Der Beitrag legt deshalb einen starken Akzent auf diese Öffentlichkeitsbeteiligung, weil der Entstehungsprozess von Regelungsinhalten bedeutsamer wird und größere Aufmerksamkeit auf sich zieht. Das schränkt hier den Raum für die Inhalte der Verordnung ein.

\section{StandAG und VO-Ermächtigungen}

Mit dem Standortauswahlverfahren soll nach $\$ 1$ Abs. 2 S. 1 in einem partizipativen, wissenschaftsbasierten, transparenten, selbsthinterfragenden und lernenden Verfahren für die im Inland verursachten hochradioaktiven Abfälle ein Standort mit der bestmöglichen Sicherheit für eine Anlage zur Endlagerung nach $\$ 9$ a Abs. 3 S. 1 des Atomgesetzes in der Bundesrepublik Deutschland ermittelt werden. Der Standort mit der bestmöglichen Sicherheit ist der Standort, der im Zuge eines vergleichenden Verfahrens aus den in der jeweiligen Phase nach den hierfür maßgeblichen Anforderungen dieses Gesetzes geeigneten Standorten bestimmt wird und die bestmögliche Sicherheit für den dauerhaften Schutz von Mensch und Umwelt vor ionisierender Strahlung und sonstigen schädlichen Wirkungen dieser Abfälle für einen Zeitraum von einer Million Jahren gewährleistet (Satz 2). ${ }^{9}$

Ein Set von Kriterien - geowissenschaftliche Kriterien nach den $\$ \$ 22$ bis 24 und die Ergebnisse der vorläufigen Sicherheitsuntersuchungen nach $\$ 27$, die Sicherheitsanforderungen nach $\$ 26$ sowie nachrangig die planungswissenschaftlichen Abwägungskriterien gem. $\ 25$ - wird auf das Territorium Deutschlands bzw. ermittelte Teilgebiete angewendet, um sukzessive den geeigneten Standort zu er-

1) GT, Der Spiegel 21/2020, S. 23.

2) Vom 6. 10.2020, BGB1. I S. 2094

3) Vgl. Smeddinck, in: Kluth/Krings (Hrsg.), Gesetzgebung, 2013, $\$ 3$ Rdnr. 73.

4) Grunwald, Physik Journal 10/2016, $20 \mathrm{ff}$.

5) Vgl. Geologiedatengesetz, Stand 19.10.2020, abrufbar unter https:// www.bundesrat.de/SharedDocs/beratungsvorgaenge/2020/0201 0300/0299-20.html;jsessionid=971B3A7C7C20A8C3D30AD34 6AF7F8D75.1_cid349? $\mathrm{nn}=47320168 \mathrm{cms}$ _topNr=299\%2F20\#top299/20.

6) Steiger, L'État, c'est moi! L'État, c'est nous! Legitimation von Staatsgewalt durch individuelle und kollektive Selbstbestimmung, in: Heschl u.a. (Hrsg.), 54. Assistententagung Öffentliches Recht „L'État, c'est quoi? Staatsgewalt im Wandel“", 2015, S. 79 ff.; Guckelberger, UTR 2011, S. $49 \mathrm{ff}$.

7) Wollenteit, NuR 2018, 668; Smeddinck, EurUP 2017, 195.

8) Hocke, Zusammenfassende Bewertung des Workshops „Sicherheitsanforderungen an die Endlagerung wärmeentwickelnder $\mathrm{Ab}$ fälle“ (Berlin, 20.+21.3.2009), Version v. 3. 9.2009 (unver.).

9) Vgl. Kersten, in: Kersten (Hrsg.), Inwastement, 2016, S. $269 \mathrm{ff}$. 
mitteln. „Die Sicherheitsanforderungen verklammern das Standortauswahlverfahren mit dem nachfolgenden Anlagenzulassungsverfahren." ${ }^{10}$ Mit der Festlegung der Kriterien vor ihrer Anwendung wird der Anspruch des StandAG, wissenschaftsbasiert und transparent zu sein, untermauert. ${ }^{11}$

Sicherheitsanforderungen sind nach $\$ 26$ Abs. 1 die Anforderungen, denen die Errichtung, der Betrieb und die Stilllegung einer nach $\$ 9 \mathrm{~b}$ Abs. 1a des Atomgesetzes genehmigungsbedürftigen Anlage zur Gewährleistung der nach dem Stand von Wissenschaft und Technik erforderlichen Vorsorge gegen Schäden genügen müssen und die damit das bei der Endlagerung zu erreichende Schutzniveau festlegen. Sie bilden die wesentliche Grundlage für die nach den $\int S 14$ (Ermittlung von Standortregionen für übertägige Erkundung), 16 (Übertägige Erkundung und Vorschlag für untertägige Erkundung) und 18 (Untertägige Erkundung) im Rahmen der vorläufigen Sicherheitsuntersuchungen nach $\$ 27$ durchzuführende Bewertung, ob an einem Standort in Verbindung mit dem vorgesehenen Endlagerkonzept der sichere Einschluss der radioaktiven Abfälle erwartet werden kann.

Das Bundesministerium für Umwelt, Naturschutz, Bau und Reaktorsicherheit (BMU) wird ermächtigt, durch Rechtsverordnung auf Grundlage der Sicherheitsprinzipien nach Absatz 2 Sicherheitsanforderungen für die Endlagerung festzulegen ( $\$ 26$ Abs. $3 \mathrm{~S}$. 1).

Das BMU wird weiterhin ermächtigt, durch Rechtsverordnung zu bestimmen, welche Anforderungen für die Durchführung der vorläufigen Sicherheitsuntersuchungen im Standortauswahlverfahren für die Endlagerung hochradioaktiver Abfälle gelten (\$27 Abs. 6 S. 1).

In beiden Verordnungsermächtigungen ist festgehalten, dass die jeweilige Verordnung spätestens zum Zeitpunkt der Durchführung repräsentativer vorläufiger Sicherheitsuntersuchungen nach $\ 14$ Abs. $1 \mathrm{~S}$. 2 vorliegen muss $(\$ 26$ Abs. 3 S. 4, \27 Abs. 6 S. 2). Beide Verordnungen sind spätestens alle zehn Jahre zu überprüfen und, soweit erforderlich, an den Stand von Wissenschaft und Technik anzupassen $\left(\$ 26\right.$ Abs. 3 S. 5, $\$ 27$ Abs. 6 S. 3). ${ }^{12}$ Die Regelung dient einem dynamischen Grundrechtsschutz. ${ }^{13}$ Der Vorschlag, den Verordnungsgeber zu verpflichten, jährlich zur Fortentwicklung zu berichten ${ }^{14}$, erscheint auch deshalb bedenkenswert, weil so eine zu starke Pfadabhängigkeit des eingeschlagenen Weges verhindert und eine zu starke zeitliche Verzögerung durch ungebremstes Beschreiten eines Holzweges vermieden werden kann.

\section{Regelungsinhalte und Kritik aus fachlicher Perspektive}

Während die VO nach Art. 1 die Sicherheitsanforderungen des BMU von $2010^{15}$ fortschreibt, bietet die VO nach Art. 2 von Grund auf neue Regelungsinhalte. Nachdem ein erster inhaltlicher Überblick schon unter II. erfolgt ist ${ }^{16}$, soll hier kurz das Regelungsprogramm referiert werden, ehe bestimmte Regelungen beispielhaft aufgegriffen werden, für die aus fachlicher oder rechtlicher Sicht ein besonderer Erörterungsbedarf besteht. Das Kapitel mündet in ein Zwischenfazit (3).

\subsection{Endlagersicherheitsanforderungsverordnung}

\subsubsection{Regelungsprogramm}

Die Verordnung ist in sechs Abschnitte gegliedert: Abschnitt 1: Allgemeine Vorschriften ( $\int 1$ : Anwendungsbereich, $\$ 2$ : Begriffsbestimmungen), Abschnitt 2: Langzeitsicherheit (\$3: Bewertungen, Entwicklungen des Endlagersystems, $\mathbb{\int} 4$ : Sicherer Einschluss der radioaktiven Abfälle, \5: Integrität und Robustheit des einschlusswirksamen Gebirgsbereichs als wesentlicher Barriere, \$6: Integrität und Robustheit der technischen und geotechnischen Barrieren als wesentliche Barrieren, \$7: Dosiswerte im Bewertungszeitraum, $\int 8$ : Ausschluss sich selbst tragender Kettenreaktionen), Abschnitt 3: Erkundung des Endlagerstandortes und Planung des Endlagers (\$ 9: Erkundung des Endlagerstandortes, \10: Sicherheitskonzept, \11: Auslegung des Endlagers, \$12: Optimierung des Endlagersystems), Abschnitt 4: Rückholbarkeit und Ermöglichung einer Bergung ( $\$ 13$ : Rückholbarkeit eingelagerter Endlagergebinde, \$14: Ermöglichung und Bergung eingelagerter Endlagergebinde), Abschnitt 5: Errichtung, Betrieb und Stilllegung des Endlagers ( $\$ 15$ : Errichtung des Endlagers, $\$ 16$ : Probebetrieb des Endlagers, \$17: Sicherheit während der Errichtung, des Betriebs und der Stilllegung des Endlagers, Anlagenzustände, $\$ 18$ : Einlagerung von radioaktiven Abfällen, (19: Stilllegung des Endlagers), Abschnitt 6: Weitere Vorschriften ( $\$ 20$ : Überwachung des Endlagers und seiner Umgebung, $\$ 21$ : Endlagerung von schwachund mittelradioaktiven Abfällen am selben Standort).

\subsubsection{Zur fachlichen Seite der Regelungsinhalte:} der einschlusswirksame Gebirgsbereich

$\mathrm{Zu}$ den markanten Fortentwicklungen gehört die Regelung des Begriffs „einschlusswirksamer Gebirgsbereich“ (ewG). In den Sicherheitsanforderungen des BMU an die Endlagerung wärmeentwickelnder radioaktiver Abfälle von 2010 findet sich nur knapp die Definition: ewG ,,ist der Teil des Endlagersystems, der im Zusammenwirken mit den technischen Verschlüssen (Schachtverschlüsse, Kammerabschlussbauwerke, Dammbauwerke, Versatz, ...) den Einschluss der Abfälle sicherstellt" ${ }^{17}$ Nach $\ 4$ Abs. 1 EndlSiAnfV sind die einzulagernden radioaktiven Abfälle im Endlagersystem mit dem Ziel zu konzentrieren und sicher einzuschließen, die darin enthaltenen Radionuklide mindestens im Bewertungszeitraum von der Biosphäre fernzuhalten. Die wesentlichen Barrieren beim sicheren Einschluss der radioaktiven Abfälle sind gem. $\$ 4$ Abs. 3 Nr. 1 ein oder mehrere ewG. Nach $\$ 5$ Abs. 1 ist für die zu erwartenden Entwicklungen im Bewertungszeitraum die Integrität des ewG zu prüfen, und darzustellen und seine Robustheit zu begründen. Der ewG ist unter Berücksichtigung der zu erwartenden Entwicklungen räumlich eindeutig zu definieren. Es ist zu prüfen und darzustellen, dass die für den sicheren Einschluss der radioaktiven Abfälle relevanten Eigenschaften der technischen und geotechnischen Barrieren mindestens in dem Zeitraum erhalten bleiben, in dem diese Barrieren nach dem Sicherheitskonzept erforderlich sind.

Der Arbeitskreis Auswahlverfahren Endlagerstandorte (AKEnd) hat 2002 hervorgehoben, dass es für die spätere Genehmigung eines Endlagers von großer Bedeutung ist, „dass das Auswahlverfahren für Endlagerstandorte auf die Auswahl von geologischen Gesamtsituationen abzielt, die einen möglichst guten ,Einschluss der Abfälle“ sicherstellen. Hierzu gehört, dass in grundwasserhaltigen Formationen der Einschluss in einem möglichst kleinräumigen ew $\mathrm{G}$ erfolgen und die Menge verunreinigten Wassers klein

10) Wollenteit, in: Frenz (Hrsg.), Atomrecht, StandAG, 2019, \$26 Rdnr. 4.

11) Wollenteit, in: Frenz (Hrsg.), Atomrecht, StandAG, 2019, $\$ 26$ Rdnr. 4.

12) Eine Befristung fordert Mehnert, Stellungnahme von endlagerdialog.de, BT-Umweltausschuss-Drs. 19(16)361-B, S. 10.

13) BVerfG, Beschl. v. 8.8.1978 - 2 BvL 8/77, BVerfGE 49, 89 ff.

14) Wollenteit, Rechtshilfe Gorleben - Beratung bzgl. Verordnung zu Sicherheitsanforderungen und VO zu Sicherheitsuntersuchungen v. 9.9 .2019 , S. 5 .

15) Stand 19.10.2020, abrufbar unter https://www.bmu.de/filead$\mathrm{min} / \mathrm{bmu}$-import/files/pdfs/allgemein/application/pdf/sicherheitsanforderungen_endlagerung_bf.pdf.

16) Vgl. Schneider, Gesetzgebung, 3. Aufl. 2002, Rdnr. 237.

17) S. 5; eingehend: Mbah, Bergwerk als technologisches Artefakt, ENTRIA-Arbeitsbericht 06, 2016, S. $95 \mathrm{ff}$. 
sein sollte (Begrenzung auf den Anlagenbereich). Dies muss durch eine Kombination von geologischen und technischen Barrieren möglichst zuverlässig und langfristig erreicht werden, um dem Schutzziel des Wasserrechtes genügen zu können." ${ }^{18}$

Dies wurde aufgrund der Anforderungen des Wasserhaushaltsgesetzes ${ }^{19}$ empfohlen, welches eine schädliche Verunreinigung des Grundwassers verbietet. ${ }^{20}$ Ein definierter ewG ${ }^{21}$ ermöglicht den Vergleich von potentiellen Endlagerstandorten anhand objektiv erscheinender Kriterien wie sie von der Endlager-Kommission festgelegt und in den Anlagen 1-12 zu $\$ 24$ StandAG niedergelegt wurden. Indem der ewG als Teil der Anlage definiert wird, kollidiert etwaiges verunreinigtes Grundwasser nicht mehr mit dem wasserrechtlichen Schutzgebot. ${ }^{22}$

Zwei Zeitprobleme seien angesprochen:

Um die größtmögliche Isolationswirkung des ewG $\mathrm{zu}$ erreichen, muss der Stand von Wissenschaft und Technik weiterhin einfließen. Dafür wäre zu klären, wie neue wissenschaftliche Erkenntnisse ,unabhängig auf ihre Güte und Relevanz für die Standortsuche und die Endlagererrichtung bewertet werden können. In Anbetracht wissenschaftlicher Kontroversen müssten daher Rahmenrichtlinien getroffen werden, unter welchen Umständen neue wissenschaftliche Erkenntnisse aus unterschiedlichen Disziplinen in das Standortauswahlverfahren bzw. in die Umsetzung des ewG-Konzepts eingebracht werden können und welche Konsequenzen sich daraus ergeben."23

Das Ziel des wirksamen Verschlusses eines Endlagers konfligiert allerdings ,mit der Forderung, zukünftigen Generationen gleichzeitig möglichst viele Handlungsspielräume offen zu halten und Fehlerkorrekturen zu ermöglichen. Dies führte zur Aufnahme der Option einer Rückholbarkeit in den rechtlichen Regelungen (vgl. Nr. 8.6 der Sicherheitsanforderungen von 2010). ${ }^{24}$ In $\int 2$ Nr. 3 StandAG wird die Rückholbarkeit als die geplante technische Möglichkeit zum Entfernen der eingelagerten Abfallbehälter mit radioaktiven Abfällen während der Betriebsphase des Endlagers definiert. Rückholbarkeit ist demnach nur vor Verschluss des Endlagers möglich.

\subsection{Endlagersicherheitsuntersuchungsverordnung}

\subsubsection{Regelungsprogramm}

Die Verordnung enthält kein Abschnitte: $\int 1$ : Anwendungsbereich, \$2: Begriffsbestimmungen, \$3: Untersuchungsraum, $\sqrt{ }$ 4: Allgemeine Anforderungen an die vorläufigen Sicherheitsuntersuchungen, $\ 5$ : Geosynthese, \6: Vorläufiges Sicherheitskonzept; vorläufige Auslegung des Endlagers, \$7: Analyse des Endlagersystems, \8: Betriebliche Sicherheitsanalyse, $\$ 9$ : Langzeitsicherheitsanalyse, $\$ 10$ : Umfassende Bewertung des Endlagersystems, $\$ 11: \mathrm{Be}-$ wertung von Ungewissheiten, \$12: Ableitung des Erkundungs-, Forschungs- und Entwicklungsbedarfs).

\subsubsection{Zur fachlichen Seite der Regelungsinhalte:} das Beispiel Langzeitsicherheitsanalyse

Gegenüber den Sicherheitsanforderungen des BMU von 2010 und dem dort geforderten Sicherheitsnachweis soll die entsprechende Regelung den nun erreichten Stand der fachwissenschaftlichen Diskussion repräsentieren.

Nach $\$ 9$ Abs. 1 muss die Langzeitsicherheitsanalyse den gesamten Bewertungszeitraum von einer Million Jahren ab dem vorgesehenen Verschluss des Endlagers umfassen und mindestens die folgenden Bereiche abdecken:

1. den sicheren Einschluss der radioaktiven Abfälle nach $\int 4$ EndlSiAnfV, 2. im Fall a) des $₫ 4$ Abs. 3 Nr. 1 der EndlSiAnfV die Integrität und Robustheit des ewG entsprechend $\int 5$ EndlSiAnfV oder b) des $\int 4$ Abs. 3 Nr. 2 EndlSiAnfV die Integrität und Robustheit der wesentlichen technischen und geotechnischen Barrieren entsprechend $\$ 6$ EndlSiAnfV sowie die Robustheit der weiteren Barrieren und sonstigen
Komponenten des Endlagersystems, 3. die Abschätzung der Dosiswerte nach $\$ 7$ EndlSiAnfV und 4. den Ausschluss sich selbst tragender Kettenreaktionen entsprechend $\$ 8$ EndlSiAnfV. Bei der Langzeitsicherheitsanalyse ist das Verhalten des Endlagersystems als Ganzes zu betrachten und entsprechend der zu erwartenden und der abweichenden Entwicklungen des Endlagersystems darzustellen. Absatz 2 verpflichtet dazu, für die Analyse des Verhaltens des Endlagersystems im Bewertungszeitraum hinreichend qualifizierte numerische Modellierung auf Grundlage realitätsnaher Annahmen durchzuführen. Im Verordnungsverfahren wurde der Begriff des Nachweises aus $\$ 9$ getilgt und durch Bewertung ersetzt, wohl um nicht zu vollmundig zu klingen.

Nach $\ 10$ sind in einer umfassenden Bewertung des Endlagersystems ausgehend von den Ergebnissen der Systemanalyse nach $\$ 7$ die Sicherheit des Endlagersystems sowie seine Robustheit zu bewerten. Dies umfasst insbesondere die Bewertung, inwiefern für den jeweiligen Untersuchungsraum in Verbindung mit dem zugeordneten vorläufigen Sicherheitskonzept zu erwarten ist, dass die Anforderungen an den sicheren Einschluss der radioaktiven Abfälle nach $₫ 4$ EndlSiAnfV erfüllt werden können. ${ }^{25}$

Die abschließende Sicherheitsbewertung, des Safety Cases für den letztlich ausgewählten Standort, baut auf einer umfassenden Sicherheitsanalyse auf, für die umfassende Daten und Kenntnisse über das Endlagersystem, den ewG und die geologische Umgebung erforderlich sind. ${ }^{26}$

In der einschlägigen Fachliteratur wurde die Verwendung des Begriffs Sicherheitsnachweis in den BMU-Sicherheitsanforderungen von 2010 kritisiert: Denn der Kern dessen, was eigentlich gemeint ist, die professionelle Anwendung des sog. Safety Cases als „Werkzeug zur Bewertung der Sicherheit" (Röhlig/Hocke), sei nicht vollständig getroffen: Es geht darum, Argumente und Gründe dafür vorzulegen, ein Tiefenlagerprogramm in einer bestimmten Weise fortzuführen, also eine Entscheidungsgrundlage in einem idealerweise schrittweisen und an verschiedenen Haltepunkten reversiblen Endlagerprogramm bereitzustellen." ${ }^{27}$ In der Definition der Endlagerkommission, wonach der „Safety Case“ den dokumentierten Nachweis bezeichnet, dass eine Einrichtung oder ein Produkt die vom ihm $\mathrm{zu}$ fordernden Sicherheitseigenschaften erfüllt, ${ }^{28}$ spiegelt sich dieses fortgeschrittene Verständnis noch nicht wieder. Mit der Verwendung des Begriffs „Bewertung/bewerten“ werden in der Verordnung die Sinnbedeutungen ,sprachliche Äußerung, durch die etwas, jemand bewertet wird/ dem [Geld]wert, der Qualität, Wichtigkeit nach [ein]schätzen, beurteilen" ${ }^{29}$ aktualisiert. Weitergehend wurde aus fachlicher Sicht angemahnt, ,,dass die verschiedenen Argu-

18) AKEnd Abschlussbericht 2002, S. 14

19) Heutige Fassung vom 31.7.2009, BGBl. I S. 2585; z. g. d. Ges. v. 19.6.2020, BGB1. I S. 1408.

20) Einordnend: Chaudry/Mintzlaff/Stahlmann, in: Smeddinck/ Kuppler/Chaudry (Hrsg.), Inter- und Transdisziplinarität bei der Entsorgung radioaktiver Reststoffe, 2016, S. 37, 38.

21) Grundsätzlich kritisch Mehnert, Stellungnahme von endlagerdialog.de, BT-Umweltausschuss-Drs. 19(16)361-B, S. 4 f.

22) Hinweis eines anonymen Experten.

23) Driftmann, Das Endlagerkonzept des einschlusswirksamen Gebirgsbereichs, 2017, S. 122; vgl. auch Gärditz, EurUP 2013, 2 ff.

24) Vgl. heute $\$ 26$ Abs. 3 S. 2 Nr. 2 StandAG.

25) Kritisch Driftmann, Das Endlagerkonzept des einschlusswirksamen Gebirgsbereichs, 2017, S. 133 f.

26) Röhlig/Hocke, in: Smeddinck/Kuppler/Chaudry (Hrsg.), Interund Transdisziplinarität bei der Entsorgung radioaktiver Reststoffe, 2016, S. 77, 81

27) Röhlig/Hocke, in: Smeddinck/Kuppler/Chaudry (Hrsg.), Interund Transdisziplinarität bei der Entsorgung radioaktiver Reststoffe, 2016, S. 77, 80 .

28) Kommission, Lagerung hoch radioaktiver Abfallstoffe, Abschlussbericht, K-Drs. 268, S. 54 Fn. 77.

29) Duden-Online, 19. 10.2020. 
mente und Gründe in jedem Schritt nicht nur dokumentiert, sondern ihre Geltungskraft auch in aus der Dokumentation erkennbarer Weise überprüft und ggf. revidiert werden kann." ${ }^{30}$ \&11 über die Bewertung von Ungewissheiten trägt dem nun umfassend Rechnung. Zugrunde gelegte Sachverhalte, Kenntnisdefizite, Verknüpfungen von Ungewissheiten untereinander, Ungewissheiten der Modellierung sind darzulegen, $\mathrm{zu}$ begründen und $\mathrm{zu}$ dokumentieren. Auch mögliche Erkundungs-, Forschungs- und Entwicklungsmaßnahmen sind im Hinblick auf die Zuverlässigkeit der sicherheitsgerichteten Aussagen zu bewerten.

\subsubsection{Zwischenfazit}

Auffällig ist der hohe inhaltliche Detaillierungsgrad der Ermächtigungen zu den Verordnungen, der als ungewöhnlich empfunden wird: „Meiner Meinung nach ist das StandAG im naturwissenschaftlichen Bereich schon total überreguliert - und jetzt kriegen wir eine „unterregulierte“ Verordnung dazu. " ${ }^{31}$ Das ist bemerkenswert, als ob der verfassungsrechtlich maßgebliche, aber gerade üblicherweise nicht beherzigte Wesentlichkeitsgrundsatz hier unerwartet eingelöst $\mathrm{zu}$ sein scheint. Danach muss der parlamentarische Gesetzgeber alle wesentlichen Fragen selbst entscheiden. ${ }^{32}$ Nur Materien von nachrangiger Bedeutung sind delegations- und verordnungsfähig; etwa Regelungen, die nicht die Ausübung von Grundrechten oder Angelegenheiten des Gemeinwesens betreffen. ${ }^{33}$ Im Umweltrecht entstand der generelle Eindruck, hier werde die „Wesentlichkeitstheorie“ in ihr Gegenteil verkehrt: Das Wesentliche - z.B. Grenzwerte, die trennscharf über $\mathrm{Zu}-$ lässigkeit und Unzulässigkeit eines Vorhabens entscheiden - steht in der Verordnung! ${ }^{34}$ Insofern erscheint ein viel beklagter Missstand behoben oder zumindest gemildert. Demgegenüber wurden zahlreiche Wünsche beratender Experten zu Detailregelungen im Entwurfsverfahren für die Verordnungen mit dem Hinweis abgelehnt, dass eine derartige Regelungstiefe für Verordnungen ungehörig/unzulässig sei.

Hintergrund dürfte zunächst sein, dass der Gesetzgeber im StandAG Vorarbeiten der Endlager-Kommission Rechnung tragen wollte, ${ }^{35}$ während auf der Verordnungsebene sich die professionelle, ministerielle Fachsicht durchsetzte. ${ }^{36}$ Weiterhin dürfte die bisherige Aufteilung von Regelungsgegenständen und -dichte entlang der Normenhierarchie (Gesetz, Verordnungen, Verwaltungsvorschriften) die Vorstellungswelt des BMU prägen wie sie sich auch in rechtswissenschaftlichen Konzeptionen im Sinne eines arbeitsteilig-kooperativen Regelungsverbundes widerspiegelt. ${ }^{37}$ Allerdings ist auch von einer großen (konzeptionellen) Gestaltungsoffenheit nicht nur auf Gesetzes-, sondern auch auf Verordnungsebene auszugehen. ${ }^{38}$ Fraglich ist wie mit Inhalten verfahren wird, die vollzugsrelevant sind, es aber nicht in die Verordnungen geschafft haben. Ggf. müssen die Rechtsanwender diese suchen und finden oder es wird z. B. durch die Entsorgungskommission eine entsprechende Leitlinie erarbeitet.

\section{4. Öffentlichkeitsbeteiligung beim Erlass der Verordnungen}

Inhaltliche Vorarbeiten für die Verordnungen hat die Kommission Lagerung hoch radioaktiver Abfallstoffe (sog. Endlager-Kommission) geliefert, die nach $\$ 4$ Abs. 2 Nr. 2 StandAG 2013 Vorschläge zu allgemeinen Sicherheitsanforderungen an die Lagerung insbesondere hoch radioaktiver Abfälle erarbeiten sollte. ${ }^{39}$ Auch dazu gab es eine spezifische Beteiligung von Öffentlichkeit und Experten. ${ }^{40}$ Hier wird die Thematik anhand der Facetten Dogmatische Grundlagen (1.), Vorgaben zur Öffentlichkeitsbeteiligung beim Erlass von Verordnungen (2.), Vorbereitung und Durchführung (3.), Auswirkung der Öffentlichkeitsbeteiligung auf den Verordnungstext (4.) und Öffentlichkeitsbeteiligung zwischen Legalität und Legitimität, zwischen gutem Willen und Handlungsroutinen (5.) aufgefächert.

\subsection{Dogmatische Grundlagen}

Seit einiger Zeit wird die Bedeutung der Öffentlichkeitsbeteiligung im Rahmen der Verordnungsgebung - über die Anhörung beteiligter Kreise ${ }^{41}$ hinausgehend - betont. ${ }^{42}$ Ausgangspunkt sind Überlegungen, auf diesem Wege Demokratie und Legitimation staatlichen Handelns stärken zu können. Hintergrund ist der deutliche Verlust des Vertrauens in staatliche Institutionen und Experten. ${ }^{43}$ Im Politikfeld Kernenergienutzung, Strahlenschutz, Endlagerung ist das aufgestaute Misstrauen traditionell groß. ${ }^{44}$

Nach Art. 80 Abs. 1 GG wird der Verordnungsgeber in der Exekutive (Ministerien) durch Verordnungsermächtigung im formellen Gesetz zum Erlass von Rechtsverordnungen ermächtigt. ${ }^{45}$ Damit handelt es sich lediglich um eine abgeleitete, gesetzesabhängige Rechtsetzungskompetenz, die sich nicht direkt - wie bei ,echten“ Gesetzen - aus der Verfassung ergibt. ${ }^{46}$ Dem Verordnungsverfahren fehlt die Publizität des Gesetzgebungsverfahrens. Die „Legitimationskette", die vermittelt durch die Parlamentsentscheidung für ein formales Gesetz ununterbrochen vom Volk bis $\mathrm{zu}$ den mit staatlichen Aufgaben betrauten Organen und Amtswaltern reicht,${ }^{47}$ ist hier zwar denkbar kurz.

Aber im Sog des gesellschaftlichen Wandels und der erstarkten Forderung nach Mitbestimmung ist die repräsentative Demokratie selbst in die Defensive geraten. ${ }^{48}$ So könnte die Rückbindung des Verordnungsverfahrens an eine Parlamentsentscheidung die juristische Dogmatik befriedigen. Gleiches gilt auch für ein Zustimmungserforder-

30) Röhlig/Hocke, in: Smeddinck/Kuppler/Chaudry (Hrsg.), Interund Transdisziplinarität bei der Entsorgung radioaktiver Reststoffe, 2016, S. 77, 81.

31) So die Sichtweise eines anonymen Experten.

32) Huster/Rux, in: Epping/Hillgruber (Hrsg.), Grundgesetz, 2. Aufl. 2013, Art. 20 Rdnr. 160 m.w. N.

33) Jarass, in: Jarass/Pieroth, GG, 16. Aufl. 2020, Art. 20 Rdnr. 71 ff

34) Eingehend: Böhm, Der Normmensch, 1996, S. 12f.; Reimer, in: Hoffmann-Riem/Schmidt-Aßmann/Voßkuhle (Hrsg.), Grundlagen des Verwaltungsrechts I, 2. Aufl. 2012, \$9 Rdnr. 56 m.w. N.; Reinhardt, NuR 2015, S. 289, 290 m. w. N.

35) Kommission, Lagerung hoch radioaktiver Abfallstoffe, Abschlussbericht, K-Drs. 268, S. 54, Ziff. 6.5.2.2.1 (,dass die Sicherheitsanforderungen per Bundesgesetz erlassen werden sollen."); Wollenteit, in: Frenz (Hrsg.), Atomrecht, StandAG, 2019, $\$ 26$ Rdnr. 3

36) Vgl. Tils, RuP 2002, $13 \mathrm{ff}$

37) Hill/Martini, in: Hoffmann-Riem/Schmidt-Aßmann/Voßkuhle (Hrsg.), Grundlagen des Verwaltungsrechts II, 2. Aufl. 2012, \$34 Rdnr. 25 m.w. N.

38) Vgl. Lepsius, JuS 2019, 123, 128

39) Kommission, Lagerung hoch radioaktiver Abfallstoffe, Abschlussbericht, K-Drs. 268, S. $281 \mathrm{ff}$.

40) Smeddinck/Willmann, EurUP 2014, 102, $104 \mathrm{ff}$.

41) Leitzke, Die Anhörung beteiligter Kreise nach $\$ \$ 51 \mathrm{BImSchG}$, $60 \mathrm{KrW}-/ \mathrm{AbfG}, 17$ Abs. 7 ChemG, 6 WRMG, 20 BBodSchG, 1999.

42) Vgl. Steiger, L'État, c'est moi! L'État, c'est nous! Legitimation von Staatsgewalt durch individuelle und kollektive Selbstbestimmung, in: Heschl u.a. (Hrsg.), 54. Assistententagung Öffentliches Recht „L'État, c'est quoi? Staatsgewalt im Wandel“, 2015 , S. $79 \mathrm{ff}$;; Guckelberger, UTR 2011, S. $49 \mathrm{ff}$.

43) Schnabel, Die Zeit v. 2.5.2019, S. 34; O. N., SPON v. 14.8.2019, Stand 19.3.2021, abrufbar unter https://www.spiegel.de/politik/ deutschland/matthias-platzeck-ex-spd-chef-macht-im-ostenteils-ungute-grundstimmung-aus-a-1281811.html.

44) Eingehend: Smeddinck/Mintzlaff/Pönitz, Entsorgungsforschung am Wendepunkt? 2020, S. $35 \mathrm{ff}$.

45) Voßkuhle/Wischmeyer, JuS 2015, $311 \mathrm{ff}$.

46) Uhle, Die Rechtsverordnung, in: Kluth/Krings (Hrsg.), Gesetzgebung, 2014, \$24 Rdnr. 1.

47) Krinos, Das Gesetz im demokratischen Verfassungsstaat, in Kluth/Krings (Hrsg.), Gesetzgebung, 2014, \$2 Rdnr. 75 m.w. N.

48) Blühdorn, Simulative Demokratie, 2013, S. 36; Michelsen/Walter, Unpolitische Demokratie, 2013, S. $183 \mathrm{ff}$. 
nis des Bundesrates. ${ }^{49}$ Beides erscheint aber kaum geeignet, die Kritik und das Misstrauen in der Gesellschaft zu besänftigen, geschweige denn zu befrieden. ${ }^{50}$

Misstrauische Regungen sind eher in einem Umfeld der Offenheit und Zugänglichkeit zu bezähmen. ${ }^{51} \mathrm{Ob}$ ein Verfahren zur Anhörung beteiligter Kreise unter Beteiligung der weiteren Öffentlichkeit durchgeführt werden sollte oder nicht, wird in Anbetracht heutiger Erwartungen an eine Transparenzkultur überraschenderweise keinesfalls eindeutig beantwortet. In der einschlägigen Literatur werden Pro-, aber auch Contra-Argumente genannt. So spricht gegen Transparenz und für ,,exklusive“ Beratungen unter Ausschluss der Öffentlichkeit, dass Korrekturen der eigenen Meinung und Kompromisse bei gegensätzlichen Interessen den Beteiligten leichter fallen sollen, als wenn sie unter den Augen der Öffentlichkeit agieren. ${ }^{52}$ Gerade bezogen auf frühe Stadien der Entwurfsbearbeitung wird hervorgehoben, dass der Ausschluss der Öffentlichkeit geradezu Voraussetzung sei, damit sich einzelne Beteiligte eine Meinung überhaupt erst bilden könnten und um gewisse Unsicherheiten, die naturgemäß unvermeidlich seien, der Öffentlichkeit nicht bekannt zu machen. ${ }^{53}$

Dennoch: Beteiligungsmöglichkeiten bei der Erarbeitung generell-abstrakter Normen einzuräumen, entspricht der partizipationswissenschaftlichen Anregung, bereits in einer Phase Einflussmöglichkeiten zu eröffnen und wahrzunehmen, die bisher aufgrund des abstrakten und anspruchsvollen Gegenstands und des erforderlichen Verständnisses typischerweise auch in anderen Sachbereichen gerade nicht genutzt wurden. Weiter bedeutet Handeln unter Unsicherheiten - wie z. B. bei Sicherheitsbewertungen auf dem Weg zu einem Endlager -, dass Unsicherheiten verbleiben. Das $\mathrm{zu}$ kommunizieren und in die Öffentlichkeit zu transportieren, könnte die Glaubwürdigkeit erhöhen und Misstrauen abbauen helfen. ${ }^{54} \$ 11$ EndlSiUntV über die Bewertung von Ungewissheiten weist der Sache nach beispielhaft in die richtige Richtung. Um auf die Beteiligung zurückzukommen: Wo es keine letzten Wahrheiten gibt, gibt es auch keine Gründe, irgendjemand aus dem Prozess der kollektiven Willensbildung auszuschließen. ${ }^{55}$

Die grundsätzlich fehlende Möglichkeit der Beteiligung beim Erlass von Rechtsverordnungen stellt auch generell ein Defizit dar. Der demokratische Charakter der Verordnungsgebung gehört gestärkt. ${ }^{56}$ Die Allgemeinzugänglichkeit und Neutralität von Regulierungsprozess und Regulierungsprodukt sind anzustreben. ${ }^{57}$ Die Öffentlichkeitsbeteiligung beim Erlass von Rechtsverordnungen sollte ausgeweitet werden. Nur so lässt sich in diesem Regulierungsformat eine kooperative Steuerungskultur unter Einbeziehung von Wissenschaft, Politik, Wirtschaft und Zivilgesellschaft ${ }^{58}$ realisieren. Es gibt bereits Regulierungsbeispiele. ${ }^{59}$

\subsection{Vorgaben zur Öffentlichkeitsbeteiligung beim Erlass von Verordnungen}

Das Handbuch der Rechtsförmlichkeit, das Empfehlungen zu Form und Gestaltung von Gesetzen und Rechtsverordnungen der Bundesministerien enthält und in seinen Empfehlungen auf rechtlichen Vorgaben und vor allem auf praktischen Erfahrungen aus der Rechtsetzung beruht, gibt Hinweise zu Mitwirkungsrechten bei der Verordnungsgebung. ${ }^{60}$ Nicht-staatlichen Stellen, also Privaten, können danach Mitwirkungsrechte nur in Form von Anhörungsrechten eingeräumt werden. ${ }^{61} \mathrm{Im}$ Weiteren wird auf Chancen wie Risiken verwiesen: „Die Mitwirkung Dritter bei der Verordnungsgebung kann sinnvoll sein, um besondere Ortsnähe, besonderen Sachverstand oder besondere Erfahrung für die Rechtsetzung zu nutzen. Durch Mitwirkungsrechte Dritter kann das Verfahren der Verordnungsgebung aber langwierig und fehleranfällig werden. Es sollte deshalb vor der Einräumung von Mitwirkungsrechten bei der Verordnungsgebung stets sorgfältig geprüft werden, ob diese aus besonderen Gründen für die zu erlassenden Verordnun- gen erforderlich sind." 62 Die Beteiligung Dritter bzw. der Offentlichkeit ist also nicht verpflichtend, sondern lediglich fakultativ und wird unter die Voraussetzung der gewissenhaften Prüfung der Notwendigkeit gestellt.

Wenn Mitwirkungsrechte für Dritte vorgesehen werden sollen - und das Spektrum reicht von bloßen Anhörungsrechten über Benehmens- und Einvernehmensregelungen bis zu Zustimmungsvorbehalten ${ }^{63}-$, so muss in der Ermächtigungsnorm jede Stelle, die beim Erlass der Rechtsverordnung zu beteiligen ist, genau bezeichnet und die Art ihrer Beteiligung genau angegeben werden. „Der Gesetzgeber darf sich bei der Regelung der Mitwirkung nicht auf Bestimmungen beschränken, die dem Verordnungsgeber die Entscheidung zuweisen, welche Stellen er in welchem Umfang bei der Verordnungsgebung beteiligt. Unbestimmte Sammelbezeichnungen (...) reichen regelmäßig nicht aus." 64 Nun enthalten die Verordnungsermächtigungen in $\$ \$ 26,27$ keinen Hinweis auf eine Beteiligung der Öffentlichkeit. Ein Verstoß gegen gesetzlich vorgeschriebene Mitwirkungsrechte kann zwar zur Nichtigkeit der Verordnung führen. ${ }^{65}$ Allerdings sind zum einen die Privaten bzw. die Offentlichkeit auch keine (staatliche) Stelle im Sinne des Handbuches der Rechtsförmlichkeit. Zum anderen fordert das StandAG in $\int 5$ Abs. $3 \mathrm{~S} .2$ geradezu dazu auf, zu den gesetzlich geregelten Formen der Öffentlichkeitsbeteiligung weitere informelle Formate anzubieten. Diese Maßgabe erfasst auch die Verordnungsermächtigungen.

Allerdings könnten völkerrechtliche Vorgaben nach Art. 8 Aarhus-Konvention tangiert sein. Danach muss sich jede

49) Voßkuhle/Wischmeyer, JuS 2015, 311, 312; Mann, Kommentierung Art. 80, in: Sachs (Hrsg.), Grundgesetz, 6. Aufl. 2011, Rdnr. 53 fff.

50) Eingehend: Smeddinck, in: ders./König (Hrsg.), Grenzwertbildung im Strahlenschutz, 2016, S. 81, 101.

51) Frevert, Vertrauensfragen, 2013, S. 16

52) Leitzke, Die Anhörung beteiligter Kreise nach $\$ \$ 51 \mathrm{BImSchG,}$ $60 \mathrm{KrW}-/ \mathrm{AbfG}, 17$ Abs. 7 ChemG, 6 WRMG, 20 BBodSchG, 1999, S. 121

53) Leitzke, Die Anhörung beteiligter Kreise nach $\$ \int 51 \mathrm{BImSchG}$ $60 \mathrm{KrW}-/ \mathrm{AbfG}, 17$ Abs. 7 ChemG, 6 WRMG, 20 BBodSchG, 1999, S. 122

54) Eckhardt/Rippe, Risiko und Ungewissheit, 2016, S. 154; eingehend: Grundmann, FAZ v. 3.4.2020, S. 12; vgl. dagegen auch: Mühlfried, Misstrauen, 2019.

55) Willke, Welche Expertise braucht die Politik? in: Bogner/Torgersen (Hrsg.), Wozu Experten? 2005, S. 45, 47.

56) Steiger, L'État, c'est moi! L'État, c'est nous! Legitimation von Staatsgewalt durch individuelle und kollektive Selbstbestimmung, in: Heschl u.a. (Hrsg.), 54. Assistententagung Öffentliches Recht „L'État, c'est quoi? Staatsgewalt im Wandel“", 2015, S. 79, 100.

57) Hoffmann-Riem/Fritzsche, Innovationsverantwortung - zur Einleitung, in: Eifert/Hoffmann-Riem (Hrsg.), Innovationsverantwortung, 2009, S. 11, 38.

58) Renn/Köck u. a., ZUR 2014, 281, 284

59) Smeddinck, in: ders./König (Hrsg.), Grenzwertbildung im Strahlenschutz, 2016, S. 81, 104.

60) BMJV, Handbuch der Rechtsförmlichkeit, 2008, Stand 19.3.2021, abrufbar unter https://www.bmjv.de/DE/Themen/RechtssetzungBuerokratieabbau/HDR/HDR node.html.

61) BMJV, Handbuch der Rechtsförmlichkeit, 2008, Stand 19.3.2021, abrufbar unter https://www.bmjv.de/DE/Themen/RechtssetzungBuerokratieabbau/HDR/HDR_node.html, Rdnr. 399.

62) BMJV, Handbuch der Rechtsförmlichkeit, 2008, Stand 19.3.2021, abrufbar unter https://www.bmjv.de/DE/Themen/RechtssetzungBuerokratieabbau/HDR/HDR_node.html, Rdnr. 400

63) BMJV, Handbuch der Rechtsförmlichkeit, 2008, Stand 19.3.2021, abrufbar unter https://www.bmjv.de/DE/Themen/RechtssetzungBuerokratieabbau/HDR/HDR_node.html, Rdnr. 399; vgl auch Arnstein, JAIP 1969, $216 \mathrm{ff}$.

64) BMJV, Handbuch der Rechtsförmlichkeit, 2008, Stand 19.3.2021, abrufbar unter https://www.bmjv.de/DE/Themen/RechtssetzungBuerokratieabbau/HDR/HDR node.html, Rdnr. 401.

65) BMJV, Handbuch der Rechtsförmlichkeit, 2008, Stand 19.3.2021, abrufbar unter https://www.bmjv.de/DE/Themen/RechtssetzungBuerokratieabbau/HDR/HDR_node.html, Rdnr. 399. 
Vertragspartei bemühen, zu einem passenden Zeitpunkt und solange Optionen noch offen sind, eine effektive Öffentlichkeitsbeteiligung während der durch Behörden erfolgenden Vorbereitung exekutiver Vorschriften und sonstiger allgemein anwendbarer rechtsverbindlicher Bestimmungen, die eine erhebliche Auswirkung auf die Umwelt haben können, zu fördern. Dabei handelt es sich nur um eine schwache Form der Verpflichtung. ${ }^{66}$ Verordnungsentwürfe wurden veröffentlicht. Die Möglichkeit zur Stellungnahme wurde eingeräumt. Einzig, ob ein für eine effektive Beteiligung ausreichender Zeitrahmen gegeben war, könnte neuralgisch sein. ${ }^{67}$ Eine einmalige Anhörung, nach der sich das BMU als Reaktion nur noch mit dem geänderten oder unveränderten Wortlaut der VO im Bundesgesetzblatt äußert, erscheint im Lichte der im StandAG geforderte Dialogorientierung als zu wenig. Wohl auch deshalb hat das BMU im Anschluss an die Öffentlichkeitsbeteiligung angekündigt, dass Vertreter/innen des BMU bei der Statuskonferenz des Bundesamtes für kerntechnische Entsorgungssicherheit (BfE, heute: Bundesamt für die Sicherheit der nuklearen Entsorgung, BASE) am 14. und 15.11.2019 in Berlin noch einmal wesentliche fachliche Ergebnisse des Symposiums darstellen. Am 29. 6. 2020 hat der Ausschuss für Umwelt, Naturschutz und nukleare Sicherheit des Bundestages eine Anhörung zu zur Verordnung über Sicherheitsanforderungen und vorläufige Sicherheitsuntersuchungen für die Endlagerung hochradioaktiver Abfälle durchgeführt. ${ }^{68}$

\subsection{Vorbereitung und Durchführung}

Das damalige BfE hat die Durchführung der Öffentlichkeitsbeteiligung extern vergeben und zwar mit den Zielen, zum einen ein Konzept zu entwickeln, um zeitgleich die Öffentlichkeit sowie die notwendigen Verbände bei der Erarbeitung dieser Verordnungen zu beteiligen, und zum anderen mit dem Ziel, ein Verfahren zur Öffentlichkeitsbeteiligung zu entwickeln und durchzuführen, das den klassischen Personen- und Teilnehmerkreis einer Verbändebeteiligung so erweitert, dass verstärkt interessierte Bürgerinnen und Bürger teilnehmen. ${ }^{69}$

Bereits auf der Endlagertagung im Juni 2019 an der Ev. Akademie Loccum wurde aus der Zivilgesellschaft eingefordert, dass die Verordnungsentwürfe hinreichend rechtzeitig zur Verfügung gestellt/veröffentlicht werden müssen, um sich vorbereiten zu können. Dem kam das BMU mit einer vorgezogenen Veröffentlichung entgegen. Mit einer Presse-Mitteilung wurde auf die Veranstaltung aufmerksam gemacht. ${ }^{70}$

In einer E-Mail des BMU vom 29.8.2019 an die Angemeldeten zum Symposium ,Sicherheitsanforderungen und Sicherheitsuntersuchungen: Dialog über zwei Verordnungsentwürfe im Rahmen der Standortsuche für ein Endlager hochradioaktiver Abfälle“ am 14. bis 15.9.2019 in Berlin der Veranstaltung wurden zunächst aktuelle Informationen dazu in einem beigefügten Flyer zur Verfügung gestellt. Außerdem wurde die Möglichkeit eines Online-Dialogs auf einer eigens für die Beteiligung am Verordnungsgebungsverfahren vorgehaltenen Homepage eingerichtet. Das Angebot wurde außerdem durch verschiedene aufbereitete Inhalte, z. B. eine Broschüre und ein erstes Video unterstützt, dem zwei weitere Videos folgten. ${ }^{71}$ Ferner wurde in der Mediathek der Webseite auch eine Gegenüberstellung des zur Diskussion gestellten Verordnungsentwurfs mit den Sicherheitsanforderungen des BMU aus dem Jahr 2010 verfügbar gemacht. Stellungnahmen für eine Präsentation im Rahmen des Symposiums konnten bis zum 5.9.2019 eingereicht werden. Auch war eine Anmeldung zur Teilnahme am Symposium möglich. Es fanden sich ca. 70 Teilnehmerinnen und Teilnehmer zusammen, darunter eine Handvoll Schülerinnen.

Am ersten Tag folgte auf die knappe Präsentation des Entwurfs zur EndlSiAnfV durch das BMU Stellungnahmen der Bundesgesellschaft für Endlagerung (BGE) und der Bürgerinitiative Umweltschutz Lüchow-Dannenberg. Danach wurden in parallelen Arbeitsgruppen je eine Stunde über Langzeitsicherheit (AG 1) sowie Erkundung und Planung (Sicherheitskonzept, Optimierung ...) (AG 2) und anschlieBend über Rückholbarkeit und Bergung; schwach- und mittelradioaktive Abfälle (AG 3) und parallel über Errichtung, Betrieb und Stilllegung; Überwachung (AG 4) beraten. Ergebnisse wurden auf Schautafeln im Hauptsaal zugänglich gemacht. Abschließend fand eine Podiumsdiskussion statt. Der zweite Tag folgte diesem Muster und war der EndlSiUntV gewidmet. Stellungnahmen wurde von Privatpersonen vorgetragen. Die Arbeitsgruppen behandelten den Allgemeinen Ablauf einer vorläufigen Sicherheitsuntersuchung (AG 5), den Umgang mit Ungewissheiten (AG 6), Besonderheiten der repräsentativen Sicherheitsuntersuchung sowie Vorgehen bei der Dosisabschätzung (AG 8). Die Arbeitsgruppen konnten frei gewählt werden.

Auffällig war im Hauptsaal, jedenfalls im Gegensatz zu den Öffentlichkeitsbeteiligungsformaten im Standortauswahlverfahren, zu denen der Verordnungserlass ausdrücklich nicht gehört, die „Rückkehr“ zur Frontalbestuhlung im Saal, die ebenso konfrontativ wie hoheitlich anmutet. ${ }^{72}$

Die Veranstaltung wurde anhand von ausgefüllten Fragebögen der Teilnehmerinnen und Teilnehmern evaluiert. ${ }^{73}$

\subsection{Auswirkung der Öffentlichkeitsbeteiligung auf den Verordnungstext}

Im Anschluss an die Veranstaltung hat das BMU die dort gegebene Zusage eingelöst und eine Synopse von 126 Seiten ins Internet gestellt, die Änderungen aufgrund der Öffentlichkeitsbeteiligung mit insgesamt 374 Änderungsvorschlägen transparent macht. ${ }^{74}$ Die Übersicht über eingereichte Stellungnahmen zum Referentenentwurf mit Bemerkungen des BMU unterscheidet farblich unterlegt die Kategorien, die zeigen wie auf Hinweise und Vorschläge reagiert wurde (Übernommen; Teilweise übernommen; Bereits anderweitig abgedeckt; Nicht übernommen; Kein konkreter Änderungsvorschlag; Nicht Gegenstand der Verordnung). Quantitativ überschlägig hat das BMU 52 Vorschläge voll übernommen und 39 teilweise. Inhaltlich bereits anderweitig abgedeckt waren 96 Hinweise. Nicht übernommen wurden 69 Hinweise. Aus 67 Hinweisen konnte kein konkreter Änderungsvorschlag abgeleitet werden. 44 Hinweise wurden als außerhalb des Regelungsgegenstandes und damit außerhalb der Regelungsbefugnis des Verordnungsgebers gekennzeichnet. Damit ist noch nichts über das qualitative Gewicht der Änderungen bzw. die Gewichtigkeit abgelehnter Ände-

66) Epiney/u.a., Aarhus-Konvention, 2018, Art. 8 Rdnr. 4.

67) Wollenteit, Rechtshilfe Gorleben - Beratung bzgl. Verordnung zu Sicherheitsanforderungen und VO zu Sicherheitsuntersuchungen v. 9.9.2019, S. 2

68) Vgl. Stand 19.3.2021, abrufbar unter https://www.bundestag.de/ ausschuesse/a16_umwelt/oeffentliche_anhoerungen/oeffentliche-anhoerung-77-sitzung-sicherheitsvo-enlagerung-699520.

69) Vgl. Stand 19.3.2021, abrufbar unter https://www.competitionline.com/de/ergebnisse/331702.

70) BMU, 152/19 v. 12.9.2019, Stand 19.3.2021, abrufbar unter https://www.bmu.de/pressemitteilung/bmu-stellt-verordnungfuer-sicheres-endlager-zur-debatte/.

71) Vgl. Stand 19.3.2021, abrufbar unter https://www.dialog-endlagersicherheit.de/.

72) Vgl. Kühl, in: Kluth/Smeddinck (Hrsg.), Bürgerpartizipation neu gedacht, 2019, S. 59, 116; Smeddinck, in: Hoffmann-Riem (Hrsg.), Innovationen im Recht, 2016, S. 403, 415 ff.

73) Kritisch aus Sicht der Sozialen Bewegungen: Vortrag Silke Freitag, Bisherige Erfahrungen, Panel Öffentlichkeitsbeteiligung, Alternative Status-Konferenz Ausgestrahlt (ppt/pdf), 9. 11.2019.

74) Vgl. Stand 19.3.2021, abrufbar unter https://www.bmu.de/fileadmin/Daten_BMU/Download_PDF/Glaeserne_Gesetze/ 19._Lp/endlsianf_verordnung/Stellungnahmen/endlsianf_vo_ stn_tabelle_bf.pdf; kritisch: Mehnert, Stellungnahme von endlagerdialog.de, BT-Umweltausschuss-Drs. 19(16)361-B, S. 2. 
rungsvorschläge gesagt. Die Öffentlichkeitsbeteiligung war jedenfalls nicht wirkungslos. Außerdem finden sich in der Synopse erläuternde Hinweise in unterschiedlicher Länge, warum wie mit den Vorschlägen verfahren wurde.

Um nur beispielhaft 2 Änderungsvorschläge zum ewG aufzugreifen: Unter der Ordnungsnummer 12 wurde zu $\$ 2$ zum Thema ,Einführung des Begriffs ,risikoarmer Einschluss" der Änderungsvorschlag gemacht: ,In die Begriffsbestimmungen ist aufzunehmen und die Verordnungstexte entsprechend zu korrigieren: ,risikoarmer Einschluss - Einschluss der Radionuklide mit nur geringfügiger Freisetzung, so dass die Freisetzung nur zu einem gesellschaftlich akzeptablen Schädigungsrisiko für Mensch und Natur führen kann. Der Begriff ,risikoarmer Einschluss' ersetzt den Begriff, sicherer Einschluss', der im StandAG benutzt wird, ohne ihn in den Begriffsbestimmungen zu benennen.' Begründung: Beseitigung eines Euphemismus und Korrektur der aufgeblasenen Sprache in der Endlagergesetzgebung." Das BMU antwortet in der Kategorie „Nicht übernommen“ mit einem rechtstechnischen Einwand: „Der Begriff ,sicherer Einschluss" ist bereits im StandAG eingeführt und im Zusammenhang mit Begriffsbestimmungen zu unterschiedlichen Komponenten wie z.B. einschlusswirksamer Gebirgsbereich und Endlagersystem als zielsetzendes Eigenschaftsmerkmal genannt worden. Eine Neudefinition und/oder Modifizierung des Begriffes in einem nachgeordneten Regelwerk kann daher nicht erfolgen."

Unter Ordnungsnummer 128 wird zu $\$ 4$ Abs. 5 zum Thema „Nachweis des einschlusswirksamen Gebirgsbereichs“ der Änderungsvorschlag gemacht: „Mit der Formulierung in $\ 4$ Absatz (5),Für abweichende Entwicklungen ist nachzuweisen, dass das Endlagersystem im Nachweiszeitraum seine Funktionstüchtigkeit in ausreichendem Maße beibehält' steht die Verordnung im Widerspruch zu den Anforderungen des Standortauswahlgesetzes, einen einschlusswirksamen Gebirgsbereich nachweisen zu müssen. Zudem verletzt der Maßstab einer, ausreichenden Sicherheit ' das bislang unstreitige Sicherheitskriterium einer ,bestmöglichen Sicherheit"“" Das BMU antwortet in der Kategorie „Teilweise übernommen“: „Die Formulierung ,Funktionstüchtigkeit' sowie der unbestimmte Rechtsbegriff, in ausreichendem Maße w wrden gestrichen. $\$ 4$ Abs. 5 ist in der neuen Fassung der EndlSiAnfV nun $\$ 4$ Abs. 6. Dieser wurde dahingehend präzisiert, dass das Endlagersystem für abweichende Entwicklungen im Bewertungszeitraum seine Funktionen nach $₫ 4$ Abs. 1 bis Abs. 4 beibehalten muss."

Die beiden Beispiele sind insofern typisch, als viele Änderungsvorschläge, die - wie gezeigt $-z$. T. auch umgesetzt wurden, Klärungen zu unbestimmten Rechtsbegriffen ${ }^{75}$ betrafen. Auffällig ist mit Blick auf die Statistik weiter, dass 309 Änderungsvorschläge die EndlSiAnfV betrafen, aber nur 65 die EndlSiUntV. Die Überzahl scheint ein Indikator dafür, dass interessierte Nicht-Spezialisten ganz bestimmte „Lieblingsthemen“ haben, die sie in der Öffentlichkeitsbeteiligung einbringen: eines sind Zahlenwerte (z. B. Dosis), ein anderes sind Szenarien. Dass es zur EndlSiUntV viel weniger Kommentare gab, dürfte daran liegen, dass der Safety Case ein komplexes und schwierig $\mathrm{zu}$ vermittelndes Thema ist. ${ }^{76}$ Keinen Hinweis brachte die Öffentlichkeitsbeteiligung auf den bisher überhaupt nicht thematisierten menschlichen Faktor in der Anwendung und Umsetzung der Sicherheitskriterien. ${ }^{77}$

Das BMU hat in angemessener Weise die Vorgabe des StandAG in anderen Zusammenhängen, wonach Ergebnisse der Stellungnahmen in weiteren Verfahrensschritten $\mathrm{zu}$ berücksichtigen sind ( $\$ 7 \mathrm{Abs} .1 \mathrm{~S} .31$. HS), adaptiert. Der Verdacht, ,,berücksichtigen“" sei im Grunde folgenlos, ${ }^{78}$ bestätigt sich nicht. Die Antwortkategorie „Kein konkreter Änderungsvorschlag“ mit der Erläuterung „Der Kommentar wurde zur Kenntnis genommen. Ein Anpassungsbedarf des Verordnungsentwurfes konnte daraus nicht abgeleitet werden" verdeutlicht auch, dass sich die sachkundigen Mit- arbeiter Mühe gegeben haben, den Vorschlägen gerecht zu werden und ggf. Änderungen vorzunehmen. Dass die Änderungen im Rahmen der Zuständigkeitsbefugnisse verarbeitet werden und keine weitergehenden Mitbestimmungsbefugnisse - wie häufig beim Stand AG gefordert - eingeräumt wurden, ist rechtlich nicht $\mathrm{zu}$ beanstanden. ${ }^{79}$

\section{5 Öffentlichkeitsbeteiligung zwischen Legalität}

und Legitimität, zwischen gutem Willen und Handlungsroutinen

Während der Veranstaltung wurde mehrfach von Vertretern der Zivilgesellschaft kritisiert, dass die Zeit nicht ausreiche, um sich eine fundierte Meinung zu den Entwürfen zu bilden. ${ }^{80}$ Von Seiten des BMU wurde in Reaktion auf die eingeräumte Frist zur Vorbereitung verwiesen. Das wiederum wurde damit gekontert, dass die zeitgleiche Öffentlichkeitsbeteiligung zum Entwurf für ein Geologiedatengesetz, das ebenfalls im Zusammenhang mit dem Standortauswahlgesetz steht, die eigenen Ressourcen absorbiert habe. Da kein Entgegenkommen wahrgenommen wurde, kündigten schließlich ca. ein Dutzend der aktivsten Teilnehmer an, aus diesem Grunde die Veranstaltung zu verlassen, und setzten die Ankündigung um. Erst mit einer E-Mail vom 26. 9. 2019 konnte das BMU mitteilen, dass die Frist für Stellungnahmen um gut 4 Wochen bis 20.11.2019 verlängert wurde.

Auffällig ist von Seiten des Ministeriums wie von Bürgervertretern, dass - trotzdem sich beide betont beteiligungsfreundlich gezeigt hatten ${ }^{81}$ - eine Störung eintrat, die auch die legitimationserzeugende Wirkung der Öffentlichkeitsbeteiligung in Frage stellt. Die Qualität der Öffentlichkeitsbeteiligung wurde so als unzulänglich wahrgenommen und kommuniziert und die prekäre Legitimität ${ }^{82}$, die sie vermitteln kann, fällt auf eine mindere Stufe zurück. Eine deutliche Steigerung ließe sich aber erreichen, wenn die allgemeine Öffentlichkeit bereits in der Erarbeitung des Referentenentwurfs und vorangehende fachliche Beratungen einbezogen würden. ${ }^{83}$

In einem Rechtsrahmen, der die Bedeutung von Konsens betont (vgl. $\int 5$ Abs. 1 S. 1), reicht es nicht mehr aus, dass eine Öffentlichkeitsbeteiligung verpflichtend oder fakultativ überhaupt stattfindet - möge daran teilnehmen, wer will oder auch nicht. In der ebenfalls selbstreflexiven/selbsthinterfragenden Perspektive des StandAG, die sich aus $\delta \$ 1$ Abs. 2 und 5 Abs. 3 ergibt, wird auch die Frage relevant,

75) Erbguth/Guckelberger, Allgemeines Verwaltungsrecht, 9. Aufl. 2018, \$14 Rdnr. 26ff.; Smeddinck, DÖV 1998, $370 \mathrm{ff}$

76) Im laufenden BMWi-geförderten transdisziplinären Forschungsverbund TRANSENS ist das Teilprojekt SAFE der kollaborativen wissenschaftlichen Auseinandersetzung mit Bürgerinnen und Bürgern über den Safety Case gewidmet. Vgl. Stand 19.3.2021, abrufbar unter www.transens.de.

77) Vorüberlegungen bei Straeter, Thesenpapier TRANSENS 14.4.2020 ,,,Enabler` für eine gute Sicherheits- und Fehlerkultur im Bereich der Endlagersuche" (unveröffentlicht).

78) Eingehend zur damaligen Regelung: Smeddinck, in: ders. (Hrsg.), StandAG, 2017, $\$ 9$ Rdnr. $43 \mathrm{f}$.

79) Vgl. Smeddinck/Semper, in: Brunnengräber (Hrsg.), Problemfalle Endlager, 2016, S. 235, 246f.; Smeddinck/Roßegger, NuR 2013, 548,556

80) So auch NBG, Stellungnahme v. 24.6.2020, Stand 19.3.2021, abrufbar unter https://www.nationales-begleitgremium.de/SharedDocs/Downloads/DE/Downloads_Stellungnahmen_Positionspapiere/NBG-Stellungnahme_SicherheitsAnfor_24.6.2020.pdf; jsessionid=C351AFCD5F1F61F75A27175A12F1ECD3.1_cid331? blob $=$ publicationFile $\& v=2$, S. 2 .

81) $\overline{\mathrm{Zu}}$ strategischer oder manipulativer Kommunikation: Rosanvallon, Demokratische Legitimität, 2010, S. 20; vgl. auch Waechter, VVDStRL 2013, 499, 515

82) Rosanvallon, Demokratische Legitimität, 2010, S. 15

83) Smeddinck, in: ders./König (Hrsg.), Grenzwertbildung im Strahlenschutz, 2016, S. 81 ff.; ebenso Mehnert, Stellungnahme von endlagerdialog.de, BT-Umweltausschuss-Drs. 19(16)361-B, S. 2. 
inwieweit die Rahmenbedingungen der Öffentlichkeitsbeteiligung Beteiligung ermöglichen oder konterkarieren. Über die Partizipationsmöglichkeit hinausgehend, rückt die Frage der Partizipationsfähigkeit erst allmählich ins Bewusstsein. ${ }^{84}$ Bei den Akteuren, die jeweils aufeinandertreffen, ist das Bewusstsein unterschiedlich weit entwickelt - abhängig von Vorerfahrungen, Bildung und Handlungsroutinen. Im Zweifel oder in unerwarteten Konfliktsituationen setzen sich die bisherigen Prägungen durch. ${ }^{85}$ Im einen Fall gerät die ministerielle Terminplanung unter Druck. Im anderen Fall wird die staatliche Seite trotz aller schönen Worte und gegenteiliger Bekundungen (wieder) als obrigkeitlich und unzugänglich wahrgenommen. Aus Zuwendung wird dann schnell wieder gegenseitiges Abstoßen und Enttäuschung voneinander. Trotz aller Bemühungen um einen Neustart scheint sich (wenigstens vordergründig) das alte FreundFeind-Verhaltensmuster zu bestätigen. ${ }^{86}$

In der Veranstaltung selbst hat das BMU inhaltliche Reaktionen auf Stellungnahmen mit der Begründung abgelehnt, es fehle an der Möglichkeit, der eingehenden inhaltlichen Auseinandersetzung. Nicht-staatlichen Stellen, also Privaten, können Mitwirkungsrechte nur in Form von Anhörungsrechten eingeräumt werden.$^{87}$ Demgegenüber verlangt das StandAG in $₫ 5$ Abs. 2 S. 2 Dialogorientierung in Öffentlichkeitsbeteiligungsformaten. ${ }^{88}$ Insofern ist ein Maximum an kommunikativem Geschick gefragt, um die geweckten Erwartungen nicht kontraproduktiv zu verfehlen. Außerdem hat das StandAG Bürgerinnen und Bürger zu Mitgestaltern des Verfahrens erhoben ( $\$ 5$ Abs. 1 S. 2). Dagegen wird verstoßen, wenn sie nur wie Anzuhörende behandelt werden. ${ }^{89}$ Über die Synopse mit den Kommentaren des BMU zu den Stellungnahmen ${ }^{90}$ wurde dieser kommunikative Mangel ein Stück weit aufgefangen.

Über die rechtliche Herleitung der Gewährleistung der Partizipationsfähigkeit ${ }^{91}$ hinausgehend steht vor allem die konzeptionelle und ggf. kollaborative Aufarbeitung der Erfordernisse im Rahmen des Standortauswahlverfahrens aus. Weiterhin braucht es geschultes Personal, das in den neuen Kommunikationswelten flexibler reagieren kann. Öffentlichkeitsbeteiligung, die nicht vor allem der Informationsverarbeitung dient, vermittelt - im gelungenen Fall jedenfalls - gesellschaftlichen Zusammenhalt. ${ }^{92}$

\section{Legitimität durch Nähe - Fazit und Ausblick}

Sicherheitsanforderungen, Dogmatik und Öffentlichkeitsbeteiligung werden bezogen auf die Artikel-Verordnung nach \$S 26, 27 StandAG beleuchtet. Die Logik rechtlicher Regulierung konkurriert und kontrastiert dabei - vor dem Hintergrund krisenhafter Tendenzen der Demokratieentwicklung - mit Konzepten, Handlungslogiken und Erwartungshaltungen von Endlagerforschung und Partizipationswissenschaft. ${ }^{93}$

Die Verordnung ist keine detaillierte fachliche Anweisung. Es kann nicht alles hineingeschrieben werden. Es ist ein Rahmen, der den Anwender veranlasst, dass Gebotene zu tun. Einerseits soll die Verordnung das Gesetz von überfrachtenden Details entlasten. Andererseits sollte die Verordnung als ebenfalls abstrakt-materielles Gesetzesrecht selbst nicht mit Details überfrachtet werden. ${ }^{94}$ Dass zahlreiche Verordnungen zum Bundes-Immissionsschutzgesetz z.B. dem nicht entsprechen, hat wiederum damit zu tun, dass die Einhaltung der Regeln zu einer Verteilung der Inhalte auf weitere nachrangige Regelwerke führt, was wiederum eine andere Form der Unübersichtlichkeit nach sich zieht.

Die herkömmliche rechtswissenschaftliche Dogmatik, die keine Öffentlichkeitsbeteiligung in der Verordnungsgebung vorsieht bzw. einfordert und das Handbuch der Rechtsförmlichkeit, das eine Anhörung der Öffentlichkeit optional aber auch warnend - ermöglicht, sollte auf Öffentlichkeitsbeteiligung als Regelfall umgestellt werden. Nur so lässt sich der Eindruck vermeiden, dass (positiv konnotiert) die „Fachbruderschaft" - negativ konnotiert: eine Art unzugängliches, intransparentes „Kastenregiment“ - die Festlegungen unter Ausschluss der Öffentlichkeit unter sich ausmacht. ${ }^{95}$

Verpflichtend oder nicht, wenn eine Öffentlichkeitsbeteiligung durchgeführt wird, dürfen deren Anforderungen und Bedingtheiten wie etwa die Kommunikationsleistungen nicht als nebensächlich behandelt, sondern müssen gut umgesetzt werden, was aber nicht jedem, jeder leichtfällt. Schlechte Leistungen in dieser Disziplin drohen aber den ganzen Aufwand zu konterkarieren.

Völlig unabhängig von vorhandenen oder nicht vorhandenen rechtlichen Verpflichtungen sollte die Öffentlichkeitsbeteiligung nicht nur als Gelegenheit zur Informationsbeschaffung, ${ }^{96}$ sondern zusätzlich und viel stärker als Gelegenheit zur Begegnung und zum gemeinsamen Lösen von Problemen in einer Entwicklung gelebt werden, ${ }^{97}$ in der die Gesellschaft mehr Einfluss und Gestaltungsmöglichkeiten begehrt und sonst Delegitimierungstendenzen überhand nehmen könnten. ${ }^{98}$ Hier erscheinen die herkömmlichen Formen des parlamentarisch-administrativen Systems nicht mehr ausreichend leistungsfähig und legitimationsstiftend. ${ }^{99}$ Der Bevölkerung mehr Möglichkeiten zur Beteiligung und Mitwirkung einzuräumen, den Eindruck zu gewinnen, Kontrolle zurückzuerlangen, erscheint am ehesten geeignet, der Krise der Demokratie entgegenzuwirken. ${ }^{100}$ Das Ausweichen in Online-Formate ${ }^{101}$ - krisengetrieben durch Corona oder nicht - dürfte jedenfalls durch diesen Blickwinkel Legitimationsverluste und Konfliktverschärfungen nach sich ziehen. „Die meisten Akteure sehen (...) nach wie vor den unmittelbaren, persönlichen Diskurs als Voraussetzung für gelingende Beteiligung an." ${ }^{102}$

Die staatlichen Stellen sollten sich ein emphatischeres Verständnis von Bürgernähe anverwandeln:

84) Smeddinck, in: Kluth/Smeddinck (Hrsg.), Bürgerpartizipation neu gedacht, 2019, S. 149, 172 ff.; Häfner, Das Standortauswahlgesetz und die Anti-Atom-Bewegung, 2015, S. 13.

85) Reichard, Das Personal der legalistischen Verwaltung, in: König/u. a. (Hrsg.), Grundmuster der Verwaltungskultur, 2014, S. 47, $57 \mathrm{ff}$; Smeddinck/Tils, Normgenese und Handlungslogiken in der Ministerialverwaltung, 2002, S. $264 \mathrm{ff}$.

86) Vgl. Smeddinck, DVB1. 2019, 744, 749.

87) BMJV, Handbuch der Rechtsförmlichkeit, 2008, Stand 19.3.2021, abrufbar unter https://www.bmjv.de/DE/Themen/RechtssetzungBuerokratieabbau/HDR/HDR_node.html, Rdnr. 399; kritisch: Schink, DVB1. 2011, 1377, 1382f.

88) Eingehend: Smeddinck, in: ders. (Hrsg.), StandAG, 2017, § 9 Rdnr. 37 ff. m. w. N.

89) Vgl. Waechter, VVDStRL 2013, 499, 506.

90) Vgl. oben unter 4.

91) Vgl. nochmals Smeddinck, in: Kluth/Smeddinck (Hrsg.), Bürgerpartizipation - neu gedacht, 2019, S. 149, $172 \mathrm{ff}$.

92) Kersten/Neu/Vogel, Politik des Zusammenhalts, 2019.

93) Vgl. Smeddinck/Tils, Normgenese und Handlungslogiken in der Ministerialverwaltung, 2002, S. $259 \mathrm{ff}$.

94) Schneider, Gesetzgebung, 3. Aufl. 2002, Rdnr. 248.

95) Vgl. Smeddinck, in: ders./König (Hrsg.), Grenzwertbildung im Strahlenschutz, 2016, S. 81, 92.

96) Schneider, Gesetzgebung, 3. Aufl. 2002, Rdnr. 264.

97) Sennett, Zusammenarbeit, 4. Aufl. 2014, S. $360 \mathrm{ff}$

98) Problematisierend: Schneider, Gesetzgebung, 3. Aufl. 2002, Rdnr. 265; vgl. auch Hill/Martini, in: Hoffmann-Riem/ Schmidt-Aßmann/Voßkuhle (Hrsg.), Grundlagen des Verwaltungsrechts II, 2. Aufl. 2012, Rdnr. 87.

99) Hocke/Smeddinck, GAIA 2017, $125 \mathrm{ff}$.

100) Rosanvallon, Die gute Regierung, 2018, S. 333; vgl. auch Blühdorn, Simulative Demokratie, 2013, S. 196.

101) Vgl. den Entwurf zu Planungssicherstellungsgesetz, Stand 19.2.2021, abrufbar unter https://www.bundestag.de/dokumente/textarchiv/2020/kw20-de-planungssicherstellungsgesetz-695086; Lubahn, Die Zeit v. 28.5.2020, S. 33; Leithäuser, FAZ v. 22.5.2020, S. 4.

102) Bipar/Paust, Auswirkungen der Corona-Pandemie auf die Bürgerbeteiligung, 15.5.2020, Stand 19.3.2021, abrufbar unter https://partizipendium.de/auswirkungen-der-corona-pandemie-auf-die-buergerbeteiligung/. 
,Nähe bedeutet auch Zugänglichkeit, Aufgeschlossenheit, Empfänglichkeit für andere. Sie setzt die Begegnung auf Augenhöhe, eine Leichtigkeit der Konsultation, eine gewisse Unmittelbarkeit in der Beziehung, das Fehlen von Förmlichkeit voraus. In der Politik gilt eine Instanz als bürgernah, wenn sie sich nicht auf die Befugnisse versteift, von ihrem Podest steigt, Widerspruch und Kritik akzeptiert, zur Äußerung von Meinungen ermuntert; Wenn sie kurz gesagt, weiß, dass sie nicht auf die institutionellen Abläufe pochen kann und einen flexibleren und direkteren Umgang mit den Bürgern einführen muss." ${ }^{103}$

Der französische Demokratie-Analytiker Pierre Rosanvallon verweist damit auf Ansätze, die schon aufgegriffen wurden, ${ }^{104}$ die aber heute vor allem durch die Digitalisierungsbrille gesehen werden und damit mehr Entfremdung als Nähe und Zusammenhalt bewirken dürften. ${ }^{105}$ Auch Sicherheit kann nicht allein objektiv bewertet werden. ${ }^{106}$ Das Sicherheitsgefühl basiert auf Vertrauen, das sich insbesondere durch Nähe vermittelt. ${ }^{107}$

Schließlich ist auf die ausstehende dritte Verordnung zu Dokumentationspflichten nach $\$ 38$ StandAG zu verweisen, die in inhaltlichem Zusammenhang zu dieser Artikelverordnung steht. Auch dafür ist eine Öffentlichkeitsbeteiligung zu erwägen.

Open Access. Dieser Artikel wird unter der Creative Commons Namensnennung 4.0 International Lizenz veröffentlicht, welche die Nutzung, Vervielfältigung, Bearbeitung, Verbreitung und Wieder- gabe in jeglichem Medium und Format erlaubt, sofern Sie den/die ursprünglichen Autor(en) und die Quelle ordnungsgemäß nennen, einen Link zur Creative Commons Lizenz beifügen und angeben, ob Änderungen vorgenommen wurden.

Die in diesem Artikel enthaltenen Bilder und sonstiges Drittmaterial unterliegen ebenfalls der genannten Creative Commons Lizenz, sofern sich aus der Abbildungslegende nichts anderes ergibt. Sofern das betreffende Material nicht unter der genannten Creative Commons Lizenz steht und die betreffende Handlung nicht nach gesetzlichen Vorschriften erlaubt ist, ist für die oben aufgeführten Weiterverwendungen des Materials die Einwilligung des jeweiligen Rechteinhabers einzuholen.

Weitere Details zur Lizenz entnehmen Sie bitte der Lizenzinformation auf http://creativecommons.org/licenses/by/4.0/deed.de.

Open Access funding enabled and organized by Projekt DEAL.

103) Rosanvallon, Demokratische Legitimität, 2010, S. 250.

104) Hoffmann-Riem (Hrsg.), Bürgernahe Verwaltung? 1979

105) Jäger/Lohse/Wissuwa, FAZ v. 21.4.2020, S. 3.

106) Marti, Risikoansichten, ENTRIA-Arbeitsbericht-05, 2016, S. $36 \mathrm{f}$

107) Frevert, Vertrauensfragen, 2013, S. 211; Smeddinck, in: ders. (Hrsg.), Emotionen bei der Realisierung eines Endlagers, 2018 , S. 11, 18 f.m.w. N.; Schaaf, ,Wir müssen zu einem Wir werden", Interview mit Jutta Allmendinger und Jan Wetzel, FAS v. 8.3.2020, S. 12.

\title{
Ein Landwirtschaftsgesetz für Deutschland im Zeichen des Umweltschutzes und der Biodiversität - Zentrale Gesetzesinhalte und Finanzierung
}

\author{
Detlef Czybulka/Peter Fischer-Hüftle/Ulrich Hampicke/Wolfgang Köck/José Martinez
}

C Der/die Autor(en) 2021. Dieser Artikel ist eine Open-Access-Publikation.

Nachdem bereits in einer Abhandlung (NuR 2021, Heft 4) ${ }^{1}$ Notwendigkeit, Funktion und Leitbild eines neuen Landwirtschaftsgesetzes dargestellt wurden, behandelt der vorliegende Aufsatz die er-

\section{Prof. Dr. Detlef Czybulka, Universität Rostock,}

Vorsitzender des Deutschen Naturschutzrechtstages e. V.,

Rostock, Deutschland

Peter Fischer-Hüftle, Vors. Richter am VG a.D.,

Rechtsanwalt, Regensburg, Deutschland

Prof. Dr. Ulrich Hampicke,

ehemals Lehrstuhl für Landschaftsökonomie,

Universität Greifswald, Greifswald, Deutschland

Prof. Dr. Wolfgang Köck,

Mitglied des Sachverständigenrates für Umweltfragen (SRU),

Leiter des Departments für Umwelt- und Planungsrecht am Helmholtz-Zentrum für Umweltforschung - UFZ,

Leipzig, Deutschland

Prof. Dr. José Martinez, Geschäftsführender Direktor des Instituts für Landwirtschaftsrecht der Universität Göttingen,

Mitglied des Wissenschaftlichen Beirats für Agrarpolitik,

Ernährung und gesundheitlichen Verbraucherschutz beim BMEL

sowie Zweiter Vorsitzender der Deutschen Gesellschaft

für Agrarrecht, Göttingen, Deutschland forderlichen materiellen Regelungen, insbesondere zum Schutz der Biodiversität, sowie die Finanzierung.

1. Integration der Anforderungen von Naturschutz (Biodiversität) und Landschaftspflege

\subsection{Problemstellung}

Die landwirtschaftliche Bodennutzung beansprucht unmittelbar die Hälfte unseres Staatsgebiets. Die Auswirkungen von Dünger, Pestiziden und Ammoniak erfassen noch weitere Flächen wie Wälder, Schutzgebiete, ungenutzte Biotope, Gewässer usw. und beeinflussen erheblich den Naturhaushalt und die Biodiversität. Hinzu kommt eine - insbesondere durch Flurbereinigung herbeigeführte und in weiten Teilen Deutschlands vorherrschende - ,ausgeräumte" Landschaft. Sie ist arm an natürlichen Elementen und ungenutzten Flächen. Vor diesem Hintergrund verlangen nicht nur der Schutz von Boden, Wasser, Luft und

1) Dieser trägt den vollständigen Titel „Ein Landwirtschaftsgesetz für Deutschland im Zeichen von Umweltschutz und Biodiversität - Notwendigkeit, Funktion und Leitbild" und ist gemeint, wenn nachfolgend auf „NuR 2021, Heft 4“ verwiesen wird. 